\title{
Article \\ Quality Identification of Sauce-Flavor Liquor Based on the Tyndall Phenomenon
}

\author{
Huizi Liu ${ }^{1}$, Xuezhou Yang ${ }^{2}$, Xiaofeng Su ${ }^{3}$, Shuzhi Li ${ }^{4}$, Qiyuan Du ${ }^{1}$, Yangyang Peng ${ }^{1}$, Mingming Shi ${ }^{1}$, \\ Chunfeng Guo ${ }^{1, *}$ and Jun Zou ${ }^{1,5, *}$ \\ 1 School of Science, Shanghai Institute of Technology, Shanghai 201418, China; liu_huizi0516@163.com (H.L.); \\ duqiyuan2021@163.com (Q.D.); pyy20211129@163.com (Y.P.); mmshi@sit.edu.cn (M.S.) \\ 2 Ningbo Langer Lighting Electric Co., Ltd., Ningbo 315300, China; xuezhou1115@163.com \\ 3 Yantai Huachuang Intelligence Equipment Co., Ltd., Yantai 264001, China; xiaofeng20211115@163.com \\ 4 Shanghai Yangtze Delta Innovation Institute, Shanghai 201418, China; szhi1118@163.com \\ 5 National Semiconductor Lighting Application System Engineering Technology Research Center, \\ Shanghai 201418, China \\ * Correspondence: cfguo@sit.edu.cn (C.G.); zoujun@sit.edu.cn (J.Z.)
}

check for updates

Citation: Liu, H.; Yang, X.; Su, X.; Li, S.; Du, Q.; Peng, Y.; Shi, M.; Guo, C.;

Zou, J. Quality Identification of

Sauce-Flavor Liquor Based on the

Tyndall Phenomenon. Appl. Sci. 2022,

12,53. https://doi.org/10.3390/

app12010053

Academic Editor: Bernhard Wilhelm Roth

Received: 20 October 2021

Accepted: 26 November 2021

Published: 22 December 2021

Publisher's Note: MDPI stays neutral with regard to jurisdictional claims in published maps and institutional affiliations.

Copyright: (c) 2021 by the authors. Licensee MDPI, Basel, Switzerland. This article is an open access article distributed under the terms and conditions of the Creative Commons Attribution (CC BY) license (https:// creativecommons.org/licenses/by/ $4.0 /)$.

\begin{abstract}
There is an obvious colloid state in sauce-flavor liquor due to its unique brewing process and long storage time, which is an important quality feature of sauce-flavor. Aiming at the problems of time, cost, and the strong professionalism of the traditional quality identification method, we proposed a method to identify the quality of sauce-flavored liquor based on the Tyndall phenomenon. The influence of different wavelengths of light on the light scattering in liquor was explored, and it was concluded that the ultraviolet and blue light bands have a certain efficiency in the identification of liquor. Additionally, we analyzed the relationship between the particle size uniformity of liquor colloids and liquor quality according to the Tyndall phenomenon. We found that higher quality liquor has a brighter and lighter Tyndall path and a smaller light flooding angle due to the uniformity of the particles in it. This method can be used to achieve qualitative identification and is suitable for the identification of sauce-flavored liquor.
\end{abstract}

Keywords: sauce-flavored; quality identification; Tyndall phenomenon; optical

\section{Introduction}

Liquor is one of the oldest distilled spirits in the world, with a long history and a substantial market. Due to its immense profits, there exists a variety of inferior and fake liquor on the market [1-3]; thus, the quality identification of liquor is very important. At present, the qualitative identification of liquor is mainly sensory tasting identification, which needs certain expertise $[4,5]$. Quantitative identification mainly relies on conventional detection methods, such as chromatography and spectroscopy [6-8]. Detection technology uses gas chromatography [9], liquid chromatography [10-12], mass spectrometry [13], near infrared sensor [14], and other large instruments. Although this method can be used for quality identification of liquor, it requires the use of large-scale equipment, has a high capital cost, and it is difficult to achieve rapid identification. Therefore, there is an urgent need for a method that can quickly perform qualitative testing to fill the gap in the market.

With the deepening of research on the colloid of liquor [15-18], it has been found that the Tyndall phenomenon exists in liquor colloid. There are also related reports that the Tyndall phenomenon may exist in liquor and is closely related to the quality of the liquor. To explore the relationship between liquor quality and the Tyndall phenomenon, we proposed a new method of liquor evaluation using the Tyndall phenomenon to identify liquor quality. Additionally, we studied the relationship between the quality of liquor and the size of colloidal particles in detail.

In this paper, we prepared four samples of sauce-flavor liquor and an empty bottle control group to investigate the efficiency of light in quality identification. The samples 
were characterized by the intensity of the Tyndall phenomenon, an AFM morphology test, and a light intensity test. Section 2 introduces the theoretical basis and working principle of light identification of liquor. Section 3 introduces the material preparation and experimental process of this study. Section 4 presents the results of the AFM test and the light intensity test. Finally, concluding remarks are presented in Section 5.

\section{Principle}

The effect of colloid on a light field is summed up as the attenuation action of sol particles on the light. From the perspective of energy transmission, the process of light transmission in a colloidal solution can be summarized as the process of light absorption and scattering, which causes it to decay continuously. When light enters a substance, different phenomena, such as absorption, scattering, refraction, and reflection, will occur according to the size of the particles and the wavelength of the light $[19,20]$. The interaction of liquor colloids with light mainly causes scattering, which is reflected in the corresponding relationship between the particle size of the colloid and the wavebandof the light.

Figure 1 shows a diagram describing the principle of sauce-flavor liquor identification based on the Tyndall phenomenon. This identification system includes a light source, a liquor cup, and a light intensity detector receiving device. When the light passes through the liquor volume, it will generate the Tyndall phenomenon which can clearly be seen by the human eye in the direction of the vertical light. The light is scattered in the liquor, resulting in the Tyndall phenomenon, and then the light will be received by the light intensity detector. Liquor colloids have a distribution of large and small particles. The light entering scatters the particles in the liquor, and the scattered light continues to be scattered by other particles. The same particle is scattered by many rays of light at the same time. Therefore, this particle can be regarded as an illuminator, macroscopically, which forms a bright pathway in the liquor that is the Tyndall phenomenon [19].

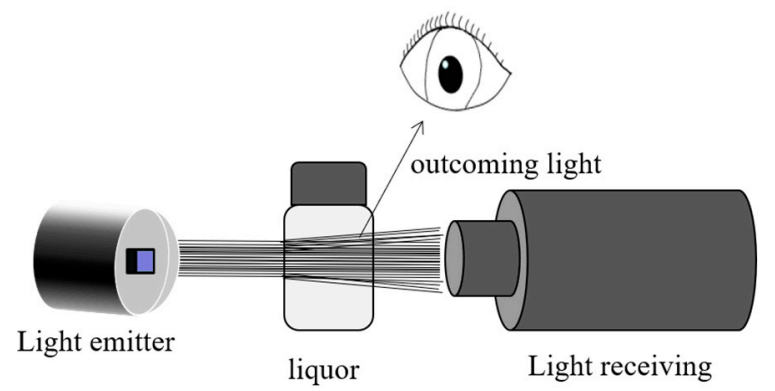

Figure 1. Principle diagram of sauce-flavor liquor identification based on the Tyndall phenomenon.

In the scattering system of liquor, because the colloidal particles move irregularly in the solution, the small particles in the solution constantly collide with sol particles that are much larger than themselves. Due to the irregularity of the collision, the sol particles that receive the impact force of the sol cannot cancel each other out, and the difference of the resultant force will result in different velocities of the colloid particles. In addition, for particles of the same size, particles with a smaller size have a larger scattering angle, and particles with a larger size have a smaller scattering angle [20]. Combining these two factors, in terms of macroscopic performance for the same incident light, if the colloidal liquor particles are small, the scattered light has a weak flood angle and its brightness is high; if the particles are large, the scattered light has a large flood angle and low center brightness. Good quality liquor has good particle size uniformity. When good quality liquor is irradiated, the light scattering tends to be produced by small particles. The light scattering angle of the particles is large, the center brightness is high, the flooding is small, and the Tyndall phenomenon shown is obvious. Poor quality liquor has poor particle size uniformity, and the light tends to be scattered by large particles. The light scattering angle of the particles is small, the center brightness is low, the flooding area is large, and the Tyndall path is dark. 


\section{Experiment}

\subsection{Material Preparation}

The lamp beads used in this experiment were 3535 spherical vertical structure lamp beads. The specific wavelength models are shown in Table 1 the liquor used was a marketable sauce-flavored liquor, purchased from a supermarket in Shanghai. Samples were numbered as sauce 1 , sauce 2 , sauce 3 , sauce 4 , and blank 1 . Among them, the difference in liquor quality between sauce 2 and sauce 3 was not substantial. Table 2 lists some basic information describing the quality of the sample liquor, which is a measure of some of the quality parameters of the liquor.

Table 1. Specifications of spherical vertical structure lamp beads.

\begin{tabular}{cc}
\hline The Serial Number & Lamp Bead Specifications (nm) \\
\hline 1 & 310 \\
2 & 340 \\
3 & 365 \\
4 & 395 \\
5 & 405 \\
6 & 440 \\
7 & 470 \\
8 & 500 \\
9 & 530 \\
10 & 560 \\
11 & 600 \\
12 & 630 \\
13 & 660 \\
\hline
\end{tabular}

Table 2. Sample parameters of sauce-flavored liquor.

\begin{tabular}{cccc}
\hline Liquor Number & Alcohol (\%vol) & Acid Value (g/L) & Total Ester (g/L) \\
\hline sauce 1 & 53 & 2.35 & 3.79 \\
sauce 2 & 53 & 2.15 & 3.25 \\
sauce 3 & 53 & 2.06 & 3.09 \\
sauce 4 & 53 & 1.56 & 2.29 \\
\hline
\end{tabular}

\subsection{Methods}

We carried out the experiments in four steps, which are as follows.

First, a darkroom experiment with light sources of all wavelengths was carried out with sauce 1 . All the lamp beads listed in Table 1 were lit up, and sauce 1 was irradiated vertically to observe the Tyndall phenomenon in the liquor.

Next, four kinds of liquor samples were tested using the lamp beads that produced obvious experimental phenomena, and the recognition ability of each light source for different liquor samples was observed.

Atomic force microscopy (AFM) (Bruker Dimension ICON, Beijing, China) was performed on the four test samples to observe their micromorphology and investigate the relationship between the quality of liquor and its micromorphology. From the observed micromorphology, we could see a difference in micromorphology and the quality of different liquor, mainly reflected in the size and uniformity of colloidal particles. The samples were diluted 3 times with deionized water, dried on a dust-free bench, and tested using the tap mode of the AFM to test the particle morphology of the different liquors. The 2D and 3D morphology and the particle size distribution of sauce-flavor liquor were observed.

Finally, a remote LED 626 light intensity test system was used to test the far-field light intensity. The light intensity from the light source was tested when passing through the empty bottles and different liquor samples. 


\section{Results and Discussion}

We selected the light source from the bands listed in Table 1, lit up 13 kinds of lamp beads in a vertical structure, and conducted the optical test on the liquor samples under dark conditions. The test liquor sample was sauce 1 . The test results showed that the liquor sample displayed an obvious Tyndall phenomenon when the light irradiated was in the ultraviolet band, but there was no obvious experimental phenomenon in visible light. The experimental results are shown in Figure 2.

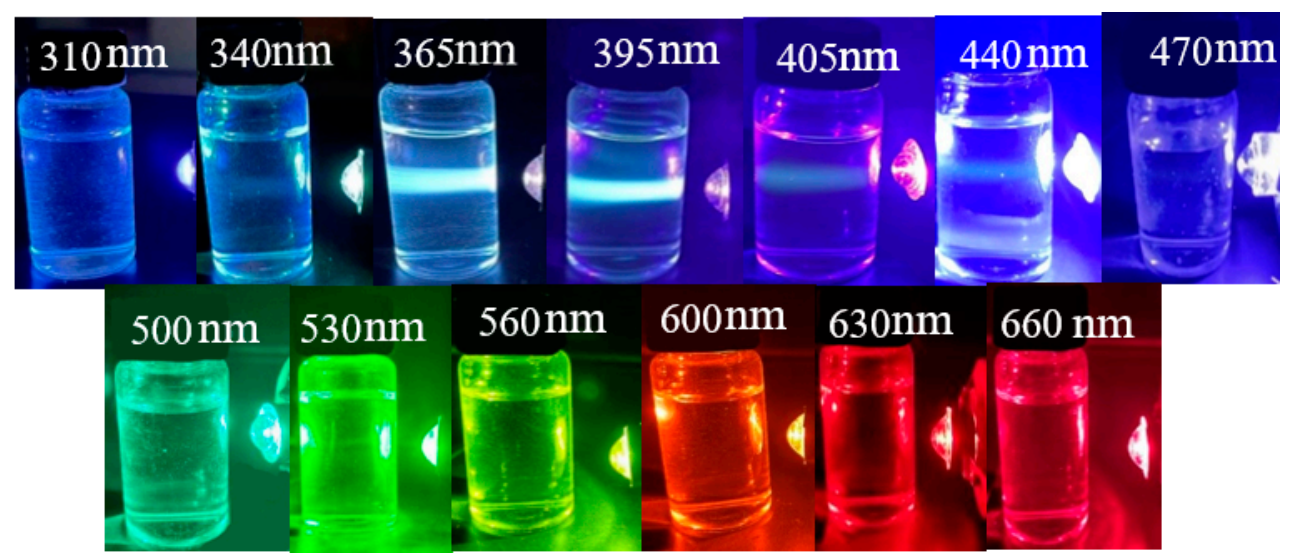

Figure 2. Phenomena of liquor under different wavelengths of light.

As can be seen from Figure 2, the Tyndall phenomenon is more obvious in the bands of $365 \mathrm{~nm}$ and $395 \mathrm{~nm}$; thus, the performance of these two lamp beads was analyzed in detail. Figure 3 shows the phenomena of the selected liquor samples in a bright environment under the irradiation of the two light beads. It can be seen that the experimental phenomena of the two lamp beads is obvious, and there is a slight difference in the visual recognition of the liquor. Therefore, we discuss the experimental phenomena of the two kinds of lamp beads in detail.
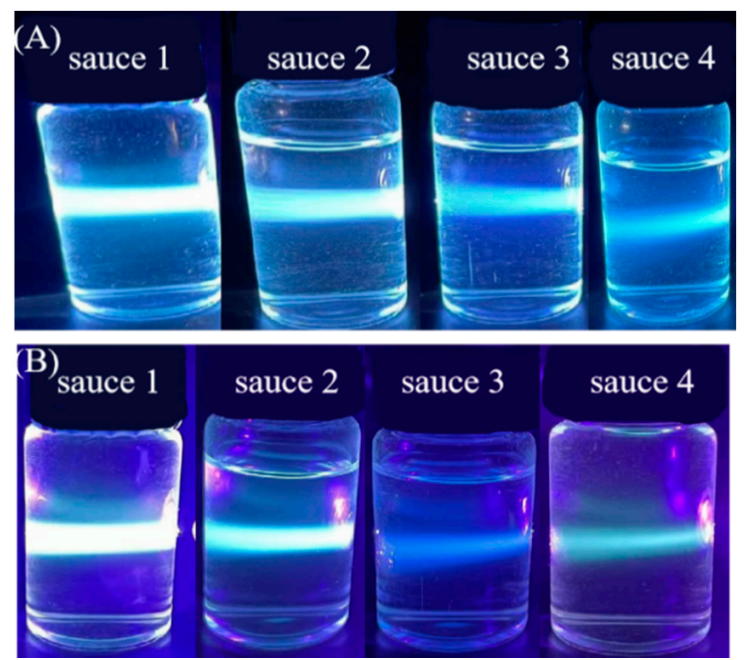

Figure 3. Sample experimental phenomena at (A) $365 \mathrm{~nm}$ and (B) $395 \mathrm{~nm}$ wavelength.

Liquor is a complex system of mixtures, often containing hundreds of aromatic substances [20]. These aromatic substances form micro-granular shapes during liquor aging. Different liquors contain different kinds and quantities of aromatic and favorable substances, and the micromorphologies formed are different [21]. Figure 4 below shows the 2D plane images and 3D morphologies of the four samples of liquor, and the height images of some large particles under the atomic force microscope. The 2D images show the particle 
size and particle distribution of the liquor colloid; the 3D images show the protuberance degree and provide a measure of the uniformity of the particles; and the height images show a quantitative analysis of the unevenness and protruding height in the 3D image. A-H refers to the special particles selected from 2D images $(a, d, g$, j), corresponding to the particle heights in height images (c, $\mathrm{f}, \mathrm{i}, \mathrm{l})$. They have a distinct pattern of identification.
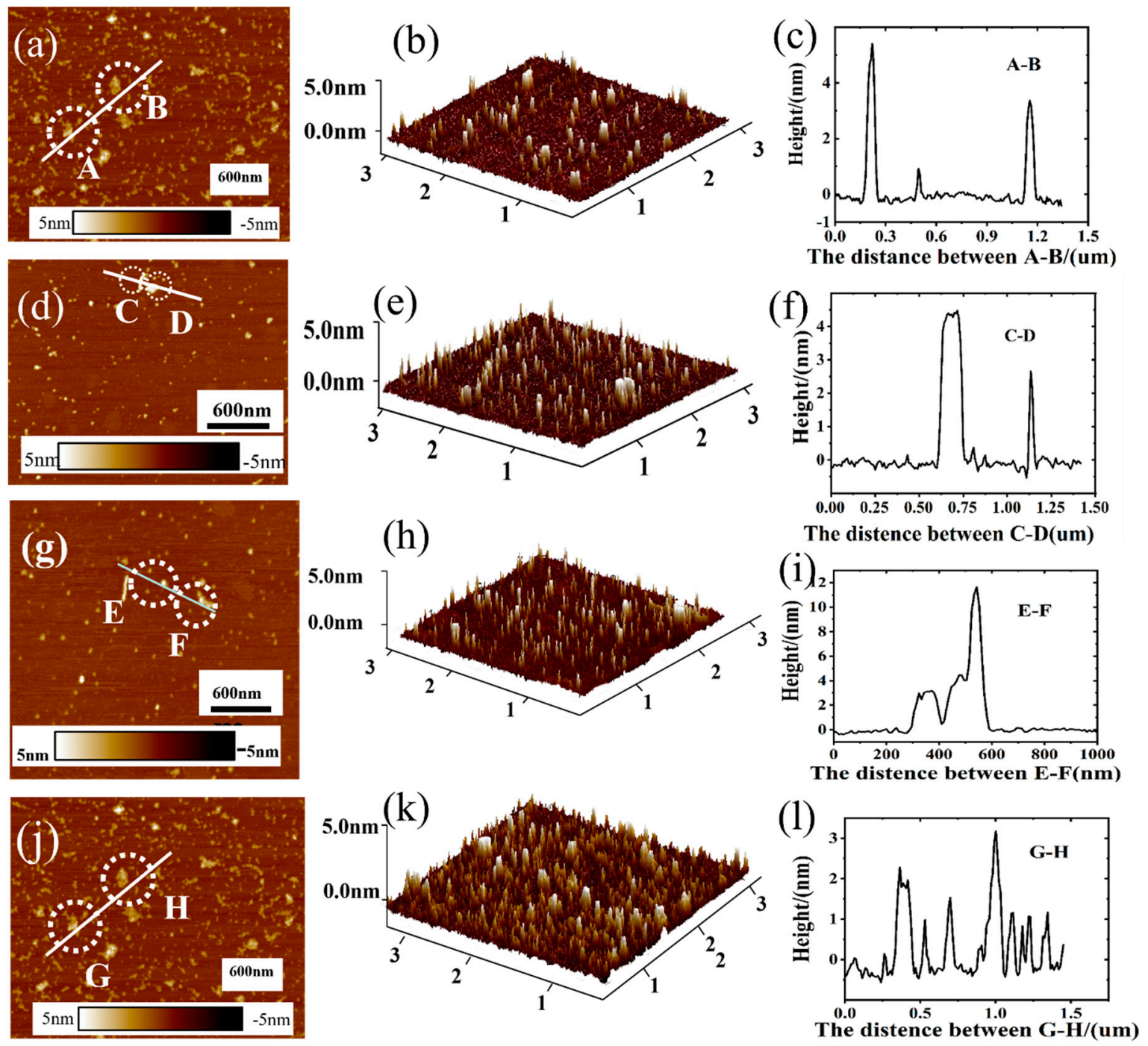

Figure 4. (a,d,g,j) 2D microscopic images of particles, (b,e,h, $\mathbf{k}) 3 \mathrm{D}$ morphology, and $(\mathbf{c}, \mathbf{f}, \mathbf{i}, \mathbf{l})$ burr height for each of the four liquor samples.

When considering the 2D plane images of the four liquor samples, Figure $4 \mathrm{a}$ is relatively uniform overall and there is no large amount of agglomerates in the field of vision. The particles are stable and uniform in size, with an overall size between $30 \mathrm{~nm}$ and $70 \mathrm{~nm}$. In Figure $4 \mathrm{~d}, \mathrm{~g}$, the particle size distribution is gradually uneven, large particles can be seen in the field of vision, and most of the particle size distribution is between 40 and $100 \mathrm{~nm}$, although some of larger particles are around $150 \mathrm{~nm}$. Figure $4 \mathrm{j}$ shows large particles with a particle size between $50 \mathrm{~nm}$ and $150 \mathrm{~nm}$, and larger particles reaching $200 \mathrm{~nm}$, with a great difference in size. Therefore, from the perspective of microparticle size, the particle size distribution of high-quality liquor is more concentrated, the gap is not large, and the content of impurities in the liquor is also less.

The 3D images show the grain uniformity of the four liquor samples. In Figure $4 b$, most of the particles extended longitudinally to $5.5 \mathrm{~nm}$, without obvious spikes and burrs, and were relatively uniform on the whole. Figure 4e,h begin to show "mushroom-like" 
protrusions in an increasing trend, with a longitudinal height roughly below $6.5 \mathrm{~nm}$. In Figure $4 \mathrm{k}$, the overall protruding amplitude is high, with "root-like" protrusions that are thick at the bottom and sharp at the top, with a tendency to connect into a network. The overall particles are disordered and aggregated, and the longitudinal elongation height is below $5 \mathrm{~nm}$. The overall uniformity is the worst among the four samples.

For the height images, some representative particles were selected from the 2D images and used to conduct a height analysis. The size distribution of the body particles in the sauce 1 liquor is concentrated, and the uniformity is good. Therefore, in Figure 4c, the overall difference in the height image is not large. Due to two special "mushroom-like" projections, the height images in Figure $4 \mathrm{f}, \mathrm{i}$ are mainly based on these projections. Compared with other particles, the two protrusions have the characteristics of thick roots and sharp heads. This is because the liquor colloid exhibits electronegativity, causing the uncharged impurities in the colloid to attract each other and result in a clustering phenomenon. It is inferred from this that in Figure 41, the roots of the particles are interconnected, the heads are sharp, and most of the particles are network-like at the bottom and almost connected into pieces. This is because the liquor colloid contains a large amount of impurities, or the liquor is too young to be a new liquor.

Through the above analysis of particle size and uniformity, when the same beam of light passes through different liquor samples the color of the light path produced by different quality liquors may be different. It is speculated that the particle size in good quality liquor is small. As the particle size increases from small to large, the choice of light scattering by the particles also changes. Small particles in liquor produce mainly ultraviolet and a small part of purple light, with a large scattering degree, and a lighter color of the Tyndall. Large particles produce mainly purple and blue light. A small part of the purpleblue light has a large degree of scattering, and the color display of the Tyndall light path is biased toward deep purple or light blue. Therefore, these two situations can be combined to distinguish the quality of sauce-flavored liquor by color. The lighter the color of the Tyndall pathway, the better the quality of the liquor; the more purple or blue the light path is, the worse the quality of the liquor.

Liquor is a rather complex system, and the content of various substances in the system will affect the light transmittance of liquor to a certain extent, and therefore affect the Tyndall phenomenon of liquor; thus, the light intensity of liquor can be tested to identify quality. Figure 5 shows the light intensity test data from four kinds of sauce-liquor and empty bottles. It can be intuitively seen that the light intensity range of lamp beads at $395 \mathrm{~nm}$ is $5400 \mathrm{mcd}$ to $7000 \mathrm{mcd}$. The $365 \mathrm{~nm}$ lamp ranges from $5000 \mathrm{mcd}$ to $6800 \mathrm{mcd}$, with slight differences in intensity and brightness. In terms of test accuracy, in Figure 5, the scattering light intensity of the four kinds of liquor are close to each other, and the data distribution is dense. In Figure $5 b$, the scattering light intensity is properly distributed, the data are relatively separate, and the interval of each liquor sample is appropriate. The light intensity was highest in the blank control group, and the intensity of sauce 1 to sauce 4 was ranked accordingly. For each sample, the test data collected at the beginning were characterized by very large intensities. This is due to the over-bright phenomenon, which is caused by an excessive current when the lamp bead is energized. The intensity then falls and gradually rises to stabilize.

As shown in Figure 5a, the overall intensity of the $365 \mathrm{~nm}$ lamp bead is low due to its large violet light composition. In the process of identification of the four kinds of samples, the differentiation between them is low. The strength of liquor of sauce 2 and sauce 3 is almost equal, which can easily be confused in macro identification. In addition, the light intensity and the time of lighting stabilization are about 50-60 s, and the identification time is about ten seconds after lighting, which varies greatly and is not good for the judgment of the final phenomenon. In Figure $5 b$, the differentiation of each sample is good, and the liquor of different quality intervals has a certain range of variation. 

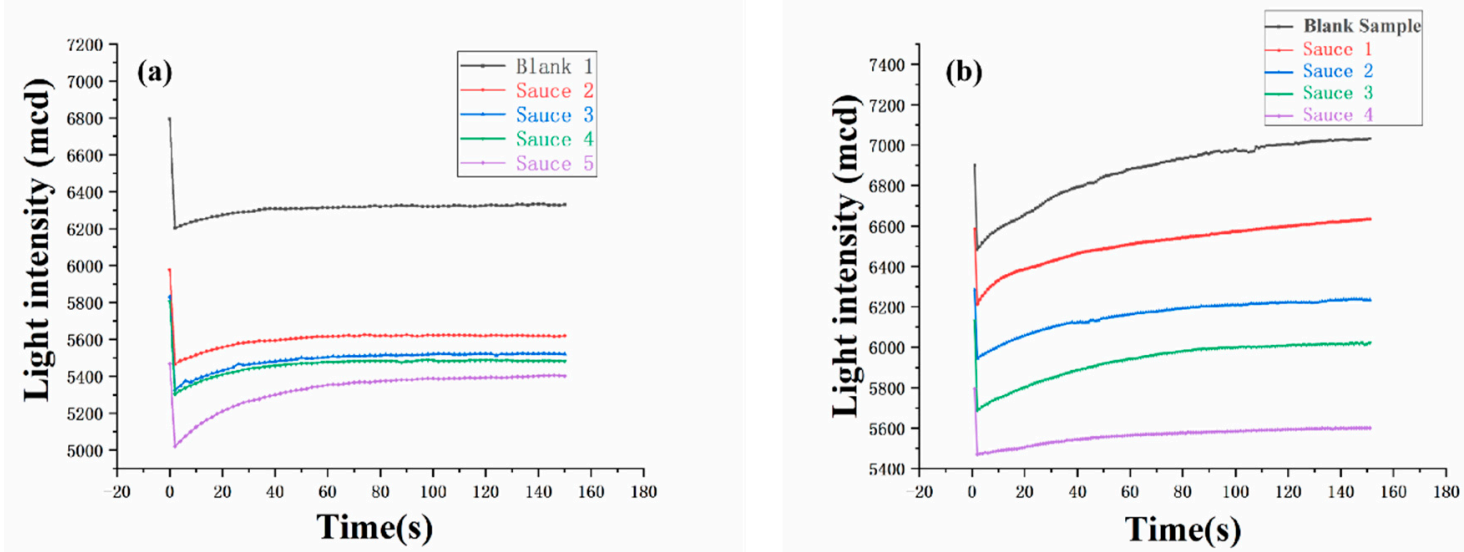

Figure 5. Time variation of the light intensity of (a) $365 \mathrm{~nm}$ and (b) $395 \mathrm{~nm}$ lamp beads after passing through different liquor samples.

\section{Conclusions}

To summarize, this paper applies an optical method to the quality identification of sauce-flavor liquor. By observing the Tyndall phenomenon of light passing through the liquor, the quality of the liquor can be comprehensively judged from the light path brightness and color shade. The results showed that the particle size and particle uniformity of the liquor colloid were the reasons for the different Tyndall phenomena. The grain size distribution of high-quality liquor was relatively uniform, and its Tyndall phenomenon was obvious. The results of liquor identification by use of our method are in good agreement with the quality of known samples. The identification of sauce-flavor liquor with our method is the first step in the food safety project, and we will continue to study the Tyndall phenomenon for food safety identification of other flavor liquor or red wine. This method has potential application value in the quality identification of sauce-flavor liquor and is suitable for industrial applications.

Author Contributions: Conceptualization, H.L., C.G. and J.Z.; methodology, J.Z.; software, Q.D.; validation, Y.P. and S.L.; formal analysis, J.Z.; investigation, M.S. and S.L.; resources, J.Z.; data curation, H.L., X.S.; writing-original draft preparation, H.L.; writing—review and editing, H.L.; visualization, H.L.; supervision, J.Z.; project administration, C.G.; funding acquisition, X.Y. All authors have read and agreed to the published version of the manuscript.

Funding: This research was funded by the Science and Technology Planning Project of Zhejiang Province, China (2018C01046), the Enterprise-funded Latitudinal Research Project (J2019-312, J2019-274, J2019-165, J2019-281, J2020-34, J2020-36, J 2020-252 and J 2020-289).

Institutional Review Board Statement: Not applicable.

Informed Consent Statement: Not applicable.

Data Availability Statement: The study did not report any data.

Acknowledgments: This work was sponsored by the Shanghai Sailing Program (18YF1422500), the Research Start-Up Project of Shanghai Institute of Technology (YJ2018-9), and the Collaborative Innovation Fund of Shanghai Institute of Technology (XTCX2019-6). Thanks to Zehe Long from Renhuai Gold Wine Co., Ltd. for his liquor samples.

Conflicts of Interest: The authors declare that they have no conflict of interest.

\section{References}

1. Zheng, Y.; Sun, B.; Zhao, M.; Zheng, F.; Huang, M.; Sun, J.; Sun, X.; Li, H. Characterization of the Key Odorants in Chinese Zhima Aroma-Type Baijiu by Gas Chromatography-Olfactometry, Quantitative Measurements, Aroma Recombination, and Omission Studies. J. Agric. Food Chem. 2016, 64, 5367-5374. [CrossRef] [PubMed]

2. Zhao, S.; Fan, W.; Yan, X.U. Study on furans in the fermented grains of sauce-flavor liquor. China Brew. $2008,21$.

3. Fan, W.L.; Xu, Y. Current practice and future trends of aroma and flavor of Chinese liquor (baijiu). J. Food Saf. Qual. 2014, 5, 3073-3078. 
4. Fan, W.; Shen, H.; Xu, Y. Quantification of volatile compounds in Chinese soy sauce aroma type liquor by stir bar sorptive extraction and gas chromatography-mass spectrometry. J. Sci. Food Agric. 2011, 91, 1187-1198. [CrossRef]

5. Li, C.; Wei, J.; Zhou, Q.; Sun, S. Rapid identification of Chinese Sauce liquor from different fermentation positions with FT-IR spectroscopy. J. Mol. Struct. 2008, 883, 99-102. [CrossRef]

6. Zhen, C.; Zhou, Y.; Zhang, N.; Wang, J.; Xiong, C.; Chen, S.; Nie, Z. Differentiation of Chinese liquors by using ambient glow discharge ionization mass spectrometry. Analyst 2013, 138, 3830-3835. [CrossRef]

7. Fan, W.; Qian, M.C. Identification of aroma compounds in Chinese 'Yanghe Daqu' liquor by normal phase chromatography fractionation followed by gas chromatography[sol ]olfactometry. Flavour Fragr. J. 2010, 21, 333-342. [CrossRef]

8. Du, H.; Xu, Y. Determination of the Microbial Origin of Geosmin in Chinese Liquor. J. Agric. Food Chem. 2012, 60, $2288-2292$. [CrossRef]

9. Sun, S.; Li, C.; Wei, J.; Zhou, Q.; Noda, I. Discrimination of Chinese Sauce liquor using FT-IR and two-dimensional correlation IR spectroscopy. J. Mol. Struct. 2006, 799, 72-76. [CrossRef]

10. Fulcrand, H.; Remy, S.; Souquet, J.; Cheynier, V.; Moutounet, M. Study of Wine Tannin Oligomers by On-Line Liquid Chromatography Electrospray Ionization Mass Spectrometry. J. Agric. Food Chem. 1999, 47, 1023-1028. [CrossRef]

11. Goldberg, D.M.; Ng, E.; Karumanchiri, A.; Yan, J.; Diamandis, E.P.; Soleas, G.J. Assay of resveratrol glucosides and isomers in wine by direct-injection high-performance liquid chromatography. J. Chromatogr. A 1995, 708, 89-98. [CrossRef]

12. Villiers, A.; Vanhoenacker, G.; Majek, P.; Sandra, P. Determination of anthocyanins in wine by direct injection liquid chromatography-diode array detection-mass spectrometry and classification of wines using discriminant analysis. J. Chromatogr. A 2004, 1054, 195-204. [CrossRef]

13. Galletti, G.C.; Antonelli, A. The potential of pyrolysis-(Methylation)/gas chromatography/mass spectrometry in the analysis of wine polyphenolics. Rapid Commun. Mass Spectrom. 2010, 7, 656-658. [CrossRef]

14. Qu, F.; Ren, D.; Wang, J.; Zhang, Z.; Lu, N.; Meng, L. An Ensemble Successive Project Algorithm for Liquor Detection Using Near Infrared Sensor. Sensors 2016, 16, 89. [CrossRef] [PubMed]

15. Zhang, J.Y.; Ma, D.-M.; Chen, J.; Wu, S.; Sun, J. The Application of DFM in the Study of Liquor Colloid. Liquor-Mak. Sci. Technol. 2009, 5, 60-64.

16. Wu, S.; Feng, Z.P.; Liu, Y.B.; He, B. Application of Scanning Probe Microscope in Liquor Study. Liquor Mak. 2009, 3, $22-24$.

17. Zhang, L.; Zhang, S.Y.; Zhao, J.S. Research on Chinese Liquor by Atomic Force Microscopy at Nm-scale(I). Liquor-Mak. Sci. Technol. 2008, 5, 39-43.

18. Zhang, L.; Zhang, S.Y.; Zhao, J.S. Research on Chinese Liquor by Atomic Force Microscopy at Nanometer-scale(II). Liquor-Mak. Sci. Technol. 2008, 3, 40-42.

19. Zhuang, M. The Properties \& Application Theories and Approaches of Collosol in Liquors. Liquor-Mak. Sci. Technol. 2002, 2, 27-30. (In Chinese)

20. Zhang, Z.; Jiang, J.; Wang, G.; Wang, H.; Liu, J.; Wang, H. Application of Two-Dimensional Correlation UV-Vis Spectroscopy in Chinese Liquor Moutai Discrimination. Am. J. Anal. Chem. 2015, 6, 395-401. [CrossRef]

21. Georgakoudi, I.; Jacobson, B.C.; Dam, J.V.; Backman, V.; Wallace, M.B.; Müller, M.G.; Zhang, Q.; Badizadegan, K.; Sun, D.; Thomas, G.A.; et al. Fluorescence, reflectance, and light-scattering spectroscopy for evaluating dysplasia in patients with Barrett's esophagus. Gastroenterology 2001, 120, 1620-1629. [CrossRef] [PubMed] 